

\section{DISCLAIMER}

This report was prepared as an account of work sponsored by an agency of the United States Government. Neither the United States Government nor any agency Thereof, nor any of their employees, makes any warranty, express or implied, or assumes any legal liability or responsibility for the accuracy, completeness, or usefulness of any information, apparatus, product, or process disclosed, or represents that its use would not infringe privately owned rights. Reference herein to any specific commercial product, process, or service by trade name, trademark, manufacturer, or otherwise does not necessarily constitute or imply its endorsement, recommendation, or favoring by the United States Government or any agency thereof. The views and opinions of authors expressed herein do not necessarily state or reflect those of the United States Government or any agency thereof. 


\section{DISCLAIMER}

Portions of this document may be illegible in electronic image products. Images are produced from the best available original document. 
Issued by Sandia Laboratories, operated for the United States Energy Research \& Development Administration by Sandia Corporation.

\section{NOTICE}

This report was prepared as an account of work sponsored by the United States Government. Neither the United States nor the United States Energy Research \& Development Administration, nor any of their employees, nor any of their contractors, subcontractors, or their employees, makes any warranty, express or implied, or assumes any legal liability or responsibility for the accuracy, completeness or usefulness of any information, apparatus, product or process disclosed, or represents that its use would not infringe privately owned rights.

\section{Printed in the United States of America Available from}

National Technical Information Service

U. S. Department of Commerce

5285 Port Royal Road

Springfield, VA 22161

Price: Printed Copy $\$ 4.00$; Microfiche $\$ 3.00$ 


\title{
THEORETICAL CONSIDERATIONS OF MAGNETIC CUSPS FOR ELECTRON-BEAM FUSION SYSTEMS
}

\author{
Thomas P. Wright \\ Plasma Theory Division - 5241 \\ Sandia Laboratories \\ Albuquerque, New Mexico 87115
}

April 1977

\begin{abstract}
Single particle motion of relativistic electrons in cusp magnetic
\end{abstract} fields has been studied analytically and numerically to evaluate some aspects of this approach to beam transport and trapping in inertial confinement schemes. It is found that angular momentum is a serious problem, and that at least one mirror loss cone exists for any initial injection conditions. Specular and randomizing reflections are investigated. It is found that charge and current neutral injection into a cusp will not provide good energy deposition for large aspect ratios (injection radius/target radius). Experimental results reported to date are analyzed using the information obtained from this theoretical study. The aspect ratio was small enough that angular momentum limits were not reached.
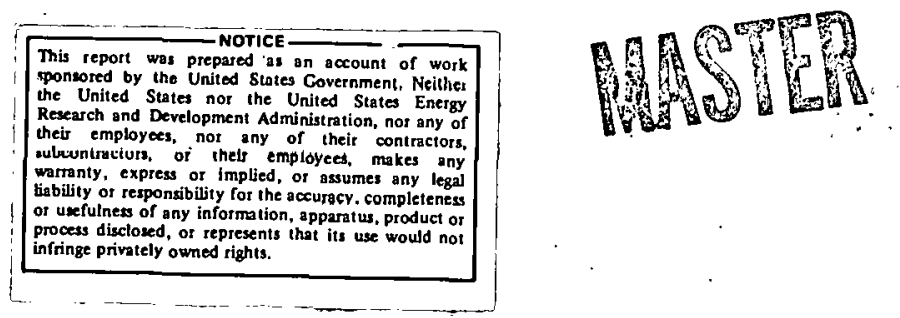


\section{TABLE OF CONTENTS}

Page

A. Description of Cusp Magnetic Field . . . . . . . . . 7

B. Hamiltonian Formalism. .............. 12

C. Loss Cones and Accessible-Inaccessible Regions . . . . . 18

D. Randomizing Reflections at Mirror Points . . . . . . . . 22

E. Summary of Reported Soviet Experimental Results. . . . . . . 24

F. Analysis of the Kurchatov Results. . . . . . . . . 26

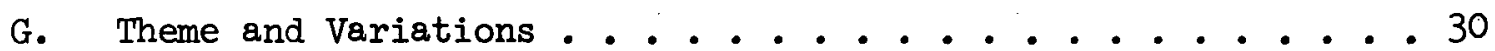

H. Conclusions. . . . . . . . . . . . . . 33

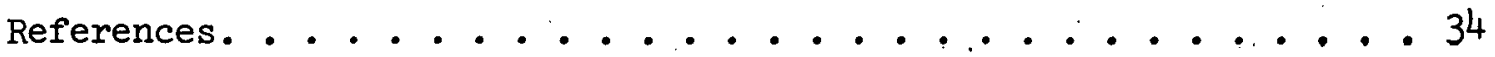


1. Contours of constant magnetic flux $\left(\mathrm{rA}_{\phi}=\right.$ constant) .... 9 .

2. Contours of constant $\mathrm{B}_{\mathrm{z}}$. : . . . . . . . . . . 10

3. Contours of constant $\mathrm{B}_{r}$. . . . . . . . . . . . 11

4. Trajectory of electron injected at cusp throat.

a) Projection of orbit in the $x-y$ plane, showing off-axis behavior for $\mathrm{z}>0$, axis encircling behavior for $\mathrm{z}<0$.

b) $r-z$ representation or orbit . . . . . .... 16

5. Electron trajectory of Fig. 4 modified by specular reflection at cusp throat. Electron exits through mirror loss cone on off-axis side. Points along extremal curves are shown . . . 20

6. Electron trajectory showing exit through loss cone on axis encircling side of cusp . . . . . . . . . . . . . . .

7. Electron trajectories of Figs. $5 \mathrm{~b}$ and $6 \mathrm{~b}$ with boundaries of accessible region shown ................

8. Electron trajectory with injection at $\mathrm{z}=1 \mathrm{~mm}$ for parameters reported by Kurchatov group. Specular reflections at cusp throat and randomizing reflections at mirror planes . . . . . . . . . . . . . . . . .

9. Electron trajectory with injection at $z=0$ to minimize initial canonical angular momentum in Kurchator configuration : . . . . . . . . . . . . . . . . . . 
THEORETICAL CONSIDERATIONS OF MAGNETIC CUSPS FOR ELECTRON-BEAM FUSION SYSTEMS

Thomas P. Wright

Plasma Theory Division - 5241

\section{A. Description of Cusp Magnetic Field}

A cusp magnetic field is generated by coaxially arranging two coils of equal radius $r_{c}$ at axial positions $\pm z_{c}$, carrying equal and opposite currents. There is cylindrical symetry, with magnetic field components $\mathrm{B}_{r}$ and $\mathrm{B}_{\mathrm{z}}$ which can be derived from a vector potential:

$$
\begin{aligned}
& A_{\phi}(r, z)=\frac{\mu_{0} I}{\pi}\left(\frac{r_{c}}{r}\right)^{I / 2}\left\{\frac{I}{k_{+}}\left[\left(1-\frac{k_{+}^{2}}{2}\right) K\left(k_{+}\right)-E\left(k_{+}\right)\right]\right. \\
& \left.-\frac{1}{k_{-}}\left[\left(1-\frac{k^{2}}{2}\right) \dot{K}\left(k_{-}\right)-E\left(k_{-}\right)\right]\right\} \text {, } \\
& B_{r}(r, z)=\frac{\mu_{o} I}{4 \pi r\left(r r_{c}\right)^{I / 2}}\left\{\left(z+z_{c}\right) k_{+}\left[\frac{r_{c}^{2}+r^{2}+\left(z+z_{c}\right)^{2}}{\left(r-r_{c}\right)^{2}+\left(z+z_{c}\right)^{2}} E\left(k_{+}\right)-K\left(k_{+}\right)\right]\right. \\
& -\left(z-z_{c}\right) k_{-}\left[\frac{r_{c}^{2}+r^{2}+\left(z-z_{c}\right)^{2}}{\left(r-r_{c}\right)^{2}+\left(z-z_{c}\right)^{2}} E\left(k_{-}\right)-K\left(k_{-}\right)\right]
\end{aligned}
$$




$$
\begin{aligned}
& B_{z}(r, z)=\frac{\mu_{o} I}{4 \pi\left(r r_{c}\right)^{I / 2}}\left\{k_{+}\left[\frac{r_{c}^{2}-r^{2}-\left(z+z_{c}\right)^{2}}{\left(r-r_{c}\right)^{2}+\left(z+z_{c}\right)^{2}} E\left(k_{+}\right)+K\left(k_{+}\right)\right]\right. \\
& \left.-k_{-}\left[\frac{r_{c}^{2}-r^{2}-\left(z-z_{c}\right)^{2}}{\left(r-r_{c}\right)^{2}+\left(z-z_{c}\right)^{2}} E\left(k_{-}\right)+k\left(k_{-}\right)\right]\right\}
\end{aligned}
$$

with I the magnitude of the current flowing in one coil,

$$
k_{ \pm}^{2}=\frac{4 r r_{c}}{\left(r+r_{c}\right)^{2}+\left(z \pm z_{c}\right)^{2}},
$$

and the functions $K$ and $E$ are complete elliptic integrals of the first and second kind. Constant magnetic flux surfaces $\left(r A_{\phi}=\right.$ constant) are shown in Fig. I for a nominal $1 \mathrm{~m}$ configuration, where the z-axis is the axis of revolution. The position of the cusp coils are at $r=.81 \mathrm{~m}$, $\mathrm{z}=-.81 \mathrm{~m}$ (designated by an $\odot$ to indicate current flowing out of the page) and at $\mathrm{z}=.81 \mathrm{~m}$ (designated by an $\mathrm{x}$ to indicate current flowing into the page). The sign convention on the current flow is chosen to make. $\mathrm{B}_{\mathrm{z}} \lessgtr 0$ for $\mathrm{z} \gtrless 0$, respectively. Figure 2 shows contours of constant $\mathrm{B}_{\mathrm{z}}$, . which reverses sign across the $\mathrm{z}=0$ plane, and constant $\mathrm{B}_{r}$ contours are shown in Fig. 3. Since $B_{r}$ varilshes or the z-axis and $B_{z}^{\prime}$ vanishes on the $z=0$ plane, the only field free region in the cusp 1 s at the origin $(r=0, z=0)$. Throughout this report the "cusp throat" will. be the neighborhood of $\left(r=r_{c}, z=0\right)$, and the mirror régions will be the neighborhoods of $\left(r=0, z= \pm z_{c}\right)$. 


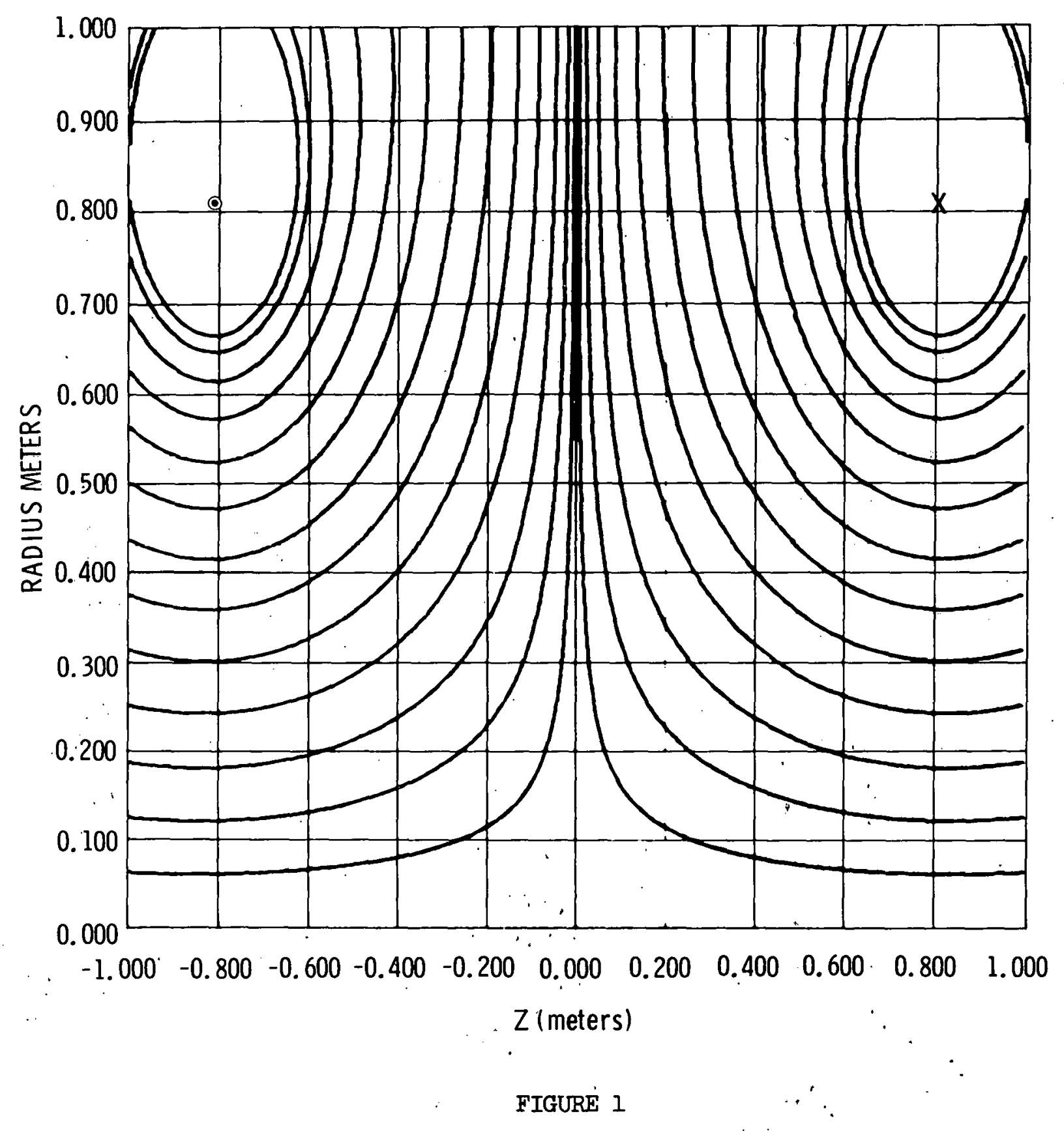




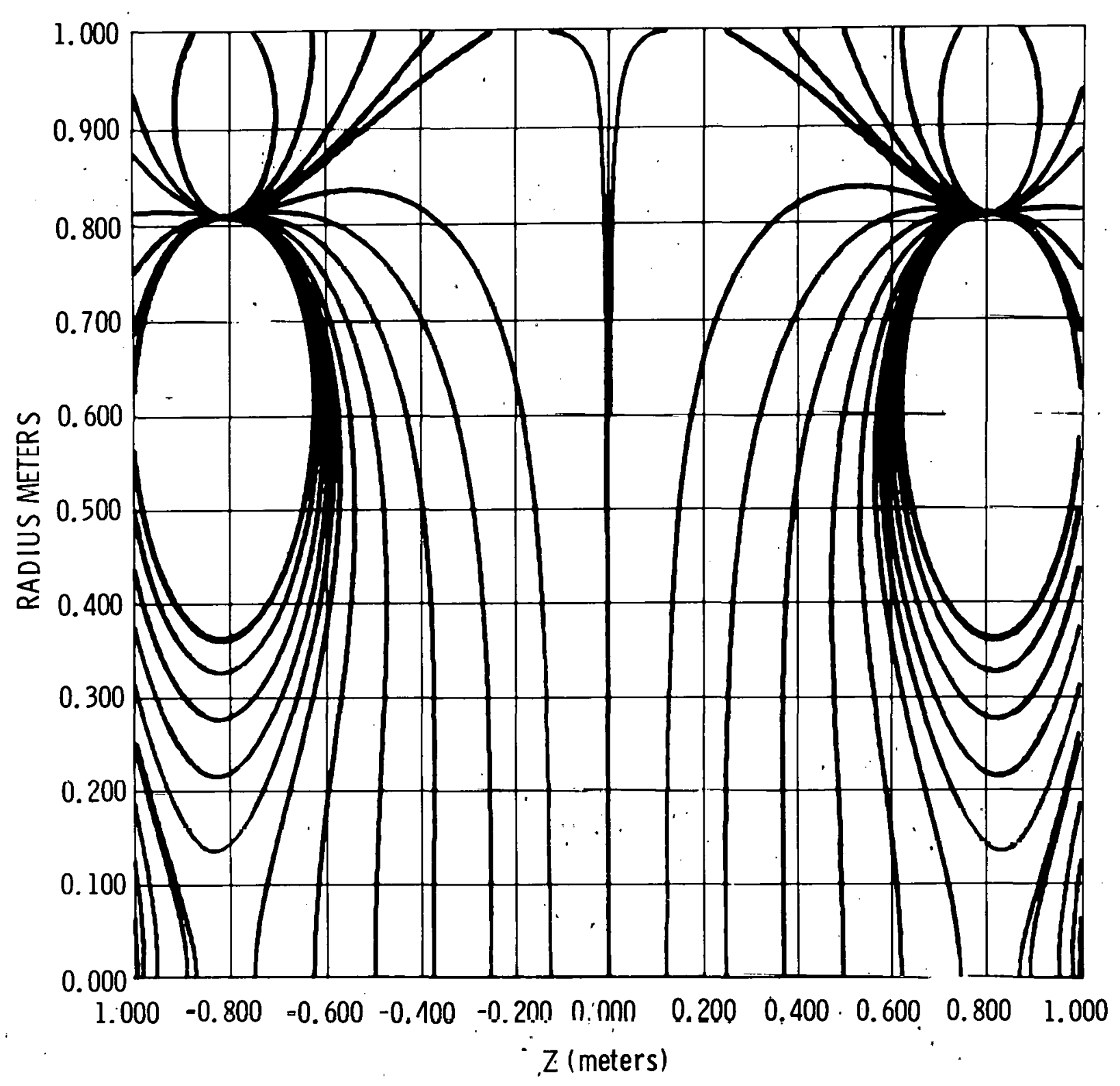

FIGURE 2 


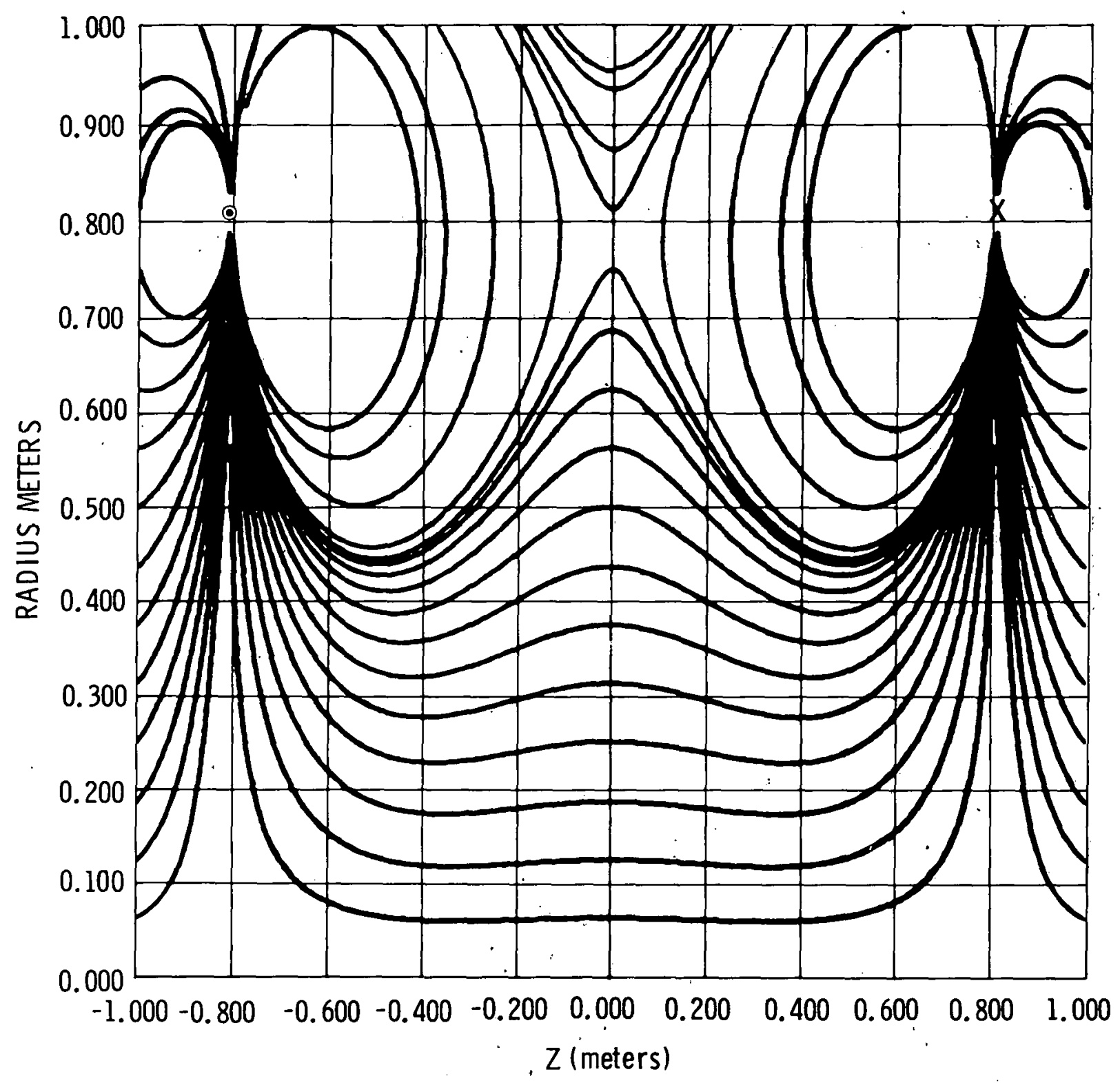

FIGURE 3 


\section{B. Hamiltonian Formalism}

Under the assumptions that an electron beam is injected into a current and charge neutralizing medium, single particle orbits are sufficient to study electron motion in the cusp. These assumptions will be reexamined later in the discussion of experimental results that have been reported.

Since $B_{z}$ reverses sign in a cusp configuration, a guiding center description of particle motion in the cusp is not possible--the particle motion is non-adiabatic. Historically, this has lead to the assumption that the particle "loses its memory" while moving in the cusp region, and introducing randomness into the calculation. schmidt $^{2}$ was the first to point out the error in this assumption and to give a correct treatment of particle motion in cusps. It is interesting to note that the published work of the Kurchatov group appears to use the randomness as sumption.

The relativistic generalization of Schmidt's work is given here. Since the cusp tield has cylindrical symmetry as seen in Figs: 1-3, a Lagrangian or Hamiltonian description of particle orbits shows that canonical angular momentum is conserved. Since the particle energy is also a constant, the two constants of the motion allow determination of many important features of particle motion in the cusp. The numerical solutions of the particle trajectories given in later sections will also include cases where effective collisions occur to determine their effect on the collisionless picture described here. 
The Lagrangian for a relativistic electron in a cusp field is given by

$$
I=-\frac{m c^{2}}{\varphi}-e v_{\phi} A_{\phi}
$$

with $\gamma=\left(1-v^{2} / c^{2}\right)^{-1 / 2}$ and $q_{e}=-e$.

The generalized coordinates are $r, z, \phi$, with $v_{r}=\dot{r}, \dot{v}_{\phi}=r \dot{\phi}$, $\mathrm{v}_{\mathrm{z}}=\dot{\mathrm{z}}$, and the canonical momenta are

$$
\begin{aligned}
& P_{r}=\frac{\partial L}{\partial \dot{r}}=r m v_{r}=p_{r} \\
& P_{z}=\gamma m v_{z}=p_{z} \\
& P_{\phi}=\frac{\partial L}{\partial \dot{\phi}}=r\left(\gamma m r \dot{\phi}-e A_{\phi}\right)=r\left(p_{\phi}-e A_{\phi}\right),
\end{aligned}
$$

where the respective mechanical momenta are notated as lower case characters. The Hamiltonian is given by

$$
H=P_{i} \dot{q}_{i}-L=r m c^{2}
$$

Note that the relativistic factor can also be written as $Y=\left[1+(p / m c)^{2}\right]^{1 / 2}$. Since the field is stationary, $H$ is a constant of lie motion:

$$
\dot{H}=m c^{2}(i+U) \text {, }
$$

where $U=e V_{D} / \mathrm{mc}^{2}$, with $\mathrm{V}_{\mathrm{D}}$ the diode voltage which produces the injected electrons.

The equations of motion are obtained from cither

$$
\frac{d P_{i}}{d t}=\frac{\partial L}{\partial q_{i}}: \text { or } \quad \dot{q}_{i_{0}}=\frac{\partial H}{\partial P_{i}} \quad, \quad-\dot{P}_{i}=\frac{\partial H}{\partial q_{i}} \text {. }
$$


Since $\phi$ is an ignorable coordinate, a second constant of the motion is the canonical angular momentum about the z-axis

$$
P_{\phi}=r\left(\gamma m v_{\phi}-e A_{\phi}\right)=\text { constant }
$$

Note that the magnetic flux enclosed by a circle of radius $r$ at axial position $\mathrm{z}$ is

$$
\Phi(r, z)=2 \pi r A_{\phi}(r, z)
$$

Lines of constant flux are shown in Fig. 1. The motion of a relativistic electron in this fleld can be described by an cffcctive potential obtained by squaring the Hamiltonian:

$$
\frac{p_{r}^{2}}{m}+\frac{p_{z}^{2}}{m}+\Psi(r, z)=0
$$

with

$$
\Psi=\frac{1}{m}\left(\frac{P_{\phi}}{r}+e A_{\phi}(r, z)\right)^{2}-m c^{2} U(2+U) .
$$

Examining this effective potential well gives a lot of Information avout the particle motion. The extremal curves of the polenlial are. determined from

$$
\frac{\partial \Psi}{\partial r}=\frac{2}{m}\left(\frac{P_{\phi}}{r}+e A_{\phi}\right)\left(-\frac{P_{\phi}}{r^{2}}+\frac{e \partial A_{\varphi}}{\partial r}\right)=0
$$

'L'hree cases arise, the first being the aimple case of an elect.ron injected such that $\mathrm{P}_{\phi}=0$. From Eq. (9) the potential minimum occurs on the z-axis and in the $\mathrm{z}=0$ plane, and the electron can pass through the origin. However, a negligible number of electrons can be injected from a diode such that their canonical anguilar momentum is identically zero. 
Case two occurs when only the first term in Eq. (10)

vanishes. From Eq. (6) this condition gives $p_{\phi}=0$ for an electron at the instant that its trajectory crosses this minimum potential surface, where

$$
\Psi_{\min }=-m c^{2} \mathrm{U}(2+\mathrm{U})
$$

This means that the electron orbit cannot directly encircle the axis, but that its projection on the $x-y$ plane has off-axis loops. From the condition

$$
\operatorname{er}_{m} A_{\phi}\left(r_{m}, z\right)=-P_{\phi}
$$

and Eqs. (6-7), it is seen that the minimum potential surface coincides with a surface of constant magnetic flux. For given initial phase space coordinates $\left(\vec{r}_{0}, \vec{v}_{0}\right)$ of an injected electron, it will execute this off-axis motion on one side of the cusp. If the canonical angular momentum at the initial phase space point is determined to be $P_{0}$, then the off-axis orbits, will occur on the side of the cusp where $A_{\phi}(r, z) / P_{0}$ is negative $(<0)$. This type of trajectory is illustrated in Fig. 4, where the off-axis looping occurs on the $z>0$ side of the cusp. Two additional points which should be noted about this type of orbit is that the minimum potential has a constant value; and that a mirror loss cone always exists on the off-axis side of the cusp since the minimum potential surface follows the constant flux surface right out of the cusp region. We will return to this point later:

The other extremal condition (case three) occurs when only the second expression in Eq. (10) vanishes: 

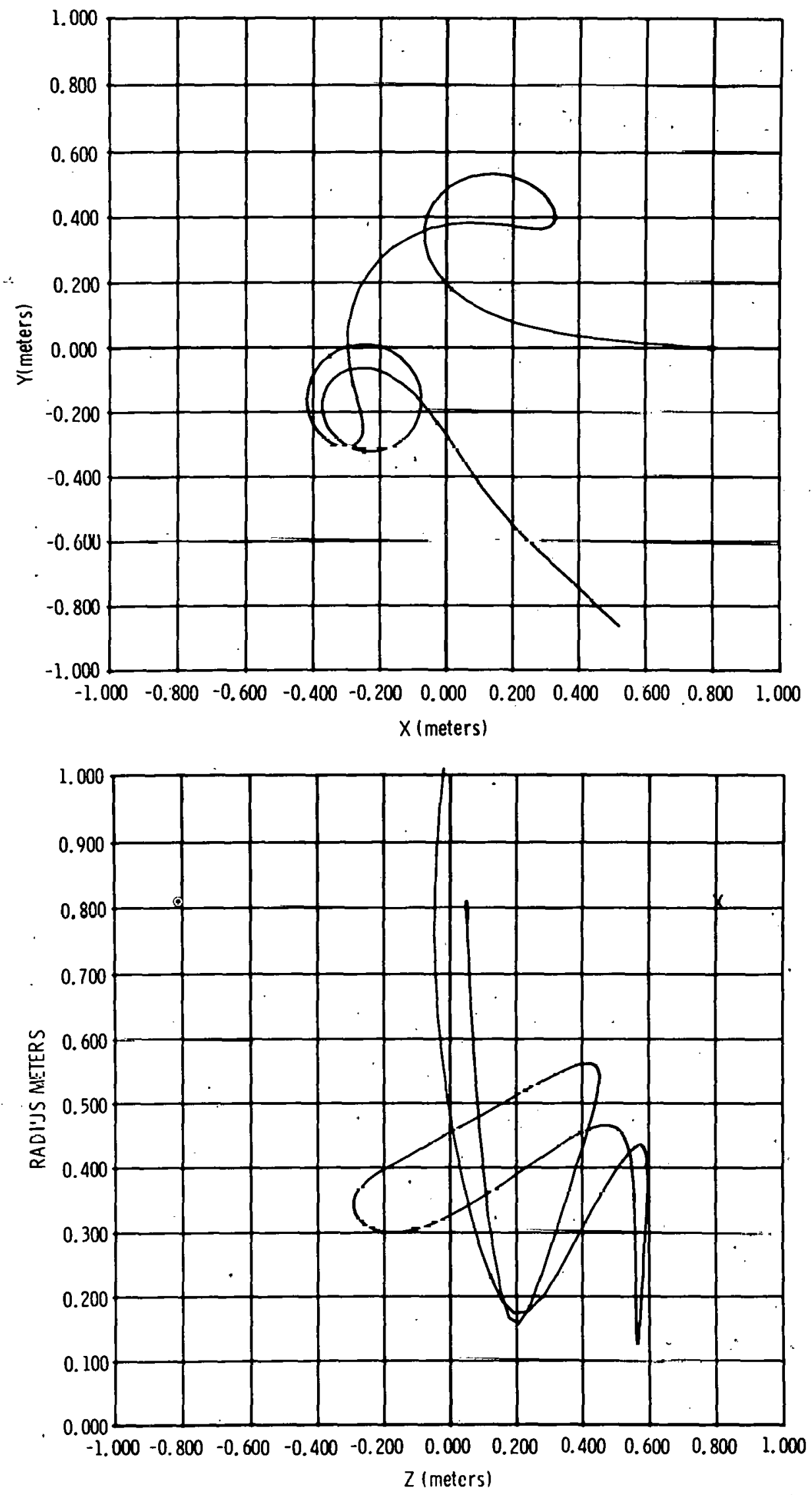

FIGURE 4 


$$
\operatorname{er}^{2} \frac{\partial A_{\phi}}{\partial r}=P_{\phi}
$$

and the value of the effective potential along this extremal surface is obtained by substitution into $\mathrm{Eq}$. (9) and using $\mathrm{B}_{z}=(1 / r) \partial\left(r A_{\phi}\right) / \partial r$ :

$$
\Psi_{M}(r, z)=\frac{e^{2}}{m} r^{2} B_{z}^{2}-m c^{2} U(2+U) \ldots
$$

In contrast to the off-axis orbits described above, here there is no point on the electron trajectory where $p_{\phi}=0$, so that on this side of the cusp (opposite the off-axis side) the electron orbit directly encircles the axis. This encircling motion occurs on the side of the cusp where $\left(A_{\phi} / P_{\phi}\right)>0$. The potential extremum of Eq. (14) can be either a maximum or minimum, and moreover, its value changes along the extremal surface. This means that in cases where it is a minimum that the effective potential well on this side of the cusp is three-dimensional, whereas the potential well on the off-axis side is two-dimensional. Therefore, depending on the particle energy, there may or may not be a mirror loss cone on the axis encircling side of the cusp. Figure 4 shows the trajectory of an electron (with nominal Hydra parameters) injected at the cusp throat, with $P_{0}>0$. Since the sign of $A_{\phi}$ is opposite to the sign of the z-coordinate according to the sign convention described above, the off-axis orbits in the $x-y$ projection shown in Fig. $4 a$ occur for $z>0$. Note that as the particle trajectory crosses the $z=0$ plane (Fig. $4 \mathrm{~b}$ ), the radius of curvature of the electron orbit (Fig. 4a) goes to infinity and then reverses as the electron orbit begins to encircle the axis. Since $\Psi_{M}$ is a maximum. 
for the electron trajectory shown, the electron is quickly ejected from the axis encircling side of the cusp across the $\mathrm{z}=0$ plane, where its radius of curvature again reverses to become an off-axis orbit. The electron then is reflected by the magnetic mirror on the $z>0$ side of the cusp (Fig. 4b) and crosses the $z=0$ plane once more as it escapes radially through the cusp throat. Note that the scales are the same in the $\mathrm{x}-\mathrm{y}$ projection with the z-axis passing through the page in the center of the grid, whereas the scales on the $r-z$ plot differ by a factor of two.

\section{Loss Cones and Accessible-Inaccessible Regions}

After reading the previous section and examining Fig. 4, an obvious question arises concerning the statement that at least one mirror loss cone exists (the one on the off-axis side), since the electron trajectory shown in Fig. 4 was clearly successfully mirrored on this side and escaped radially. To show that this loss cone really exists, and to make the calculation more relevant to reactor schemes, a specularly reflecting boundary condition is now introduced at the cusp throat to prevent radial escape. The physical mechanism which comes into play is the diode electric field which will return any radially escaping electrons to the cusp region. A specularly reflecting boundary condition assumes that anode foil scattering is negligible in repeated reflections of electrons by the diode fields. This is clearly an optimistic assumption, since any foil scattering at this large radius 
will tend to increase the electron angular momentum, thereby increasing the distance of closest approach to the z-axis. Since this is undesirable for efficient energy deposition on a target placed at the origin, the optimistic assumption is used. Since specular reflection leaves the initial constants of the motion unchanged, the extremal surfaces $\Psi_{\min }$ and $\Psi_{M}$ remain the same throughout the calculation. Figure 5 shows the resulting trajectory, with the electron escaping through the mirror loss cone. The points along $\Psi_{\min }$ and $\Psi_{\mathrm{M}}$ are obtained by numerically solving Eqs. (12) and (13) and are also plotted in Fig. 5. The extremal surfaces are obtained by rotating these lines about the z-axis. By comparison of Figs. 1 and $5 \mathrm{~b}$, it is obvious that $\Psi_{\text {min }}$ coincides with a surface of constant flux. Figure 6 shows a trajectory where $\Psi_{M}$ is a minimum and the electron escapes through the mirror loss cone on the axis encircling side of the cusp. From these considerations it is clear that it is not possible to confine an electron in a cusp without plugging the loss regions with reflecting boundaries. It has already been argued above that the optimum reflection mechanism at the cusp throat is specular reflection to avoid increasing the electron's angular momentum. If the mirror loss cones were similarly plugged, what effect would this trapping of electrons have on energy deposition on the target? The answer is very little, since angular momentum is still a factor. Since specular reflections do not change the constants of the motion, it is possible to map out the cusp region that a given electron can access using the Hamiltonian formalism of Section B. From Eq. 8, the extreme turning points of the electron trajectory in the effective potential occur at 

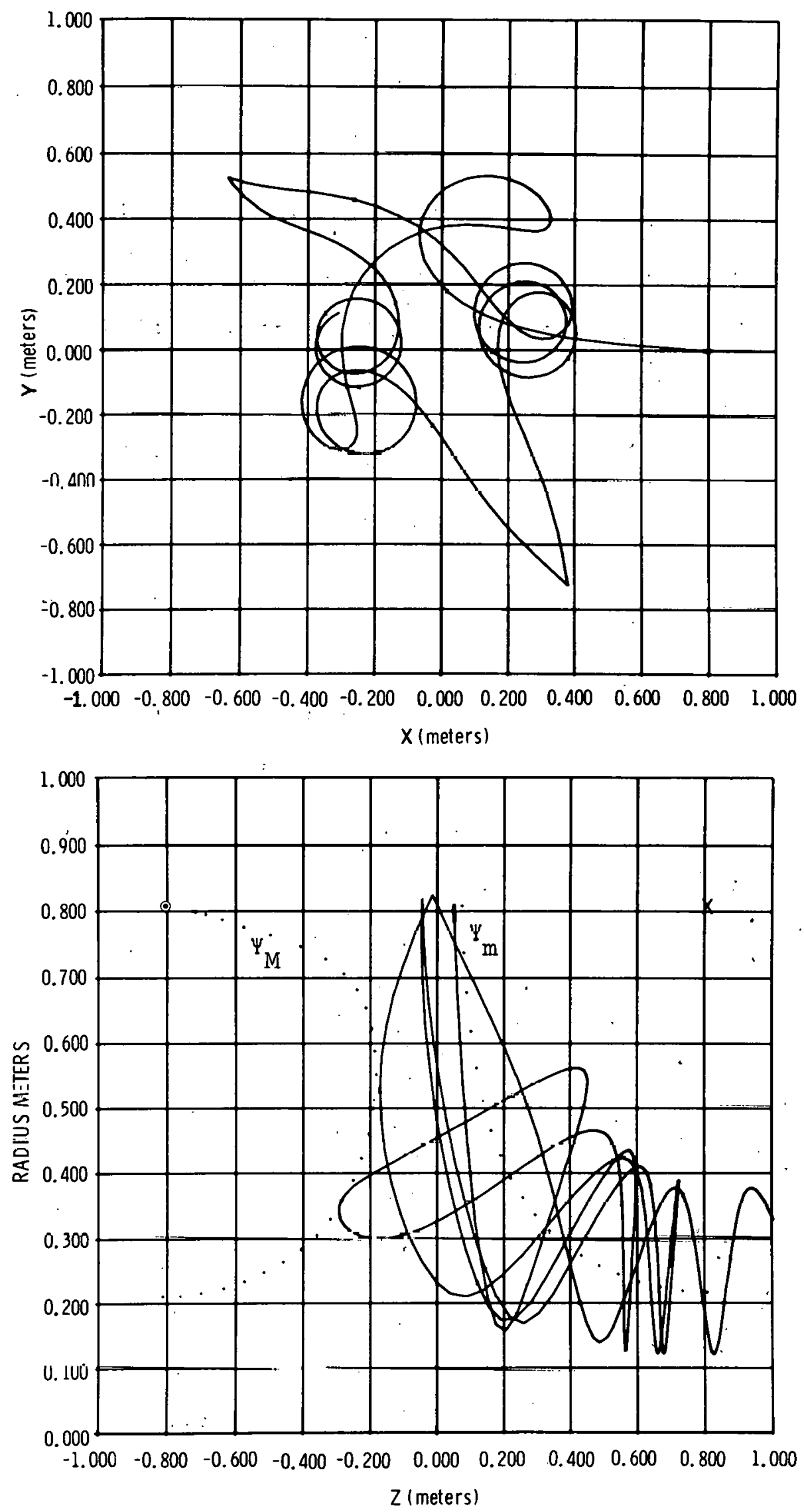

FIGURE 5 

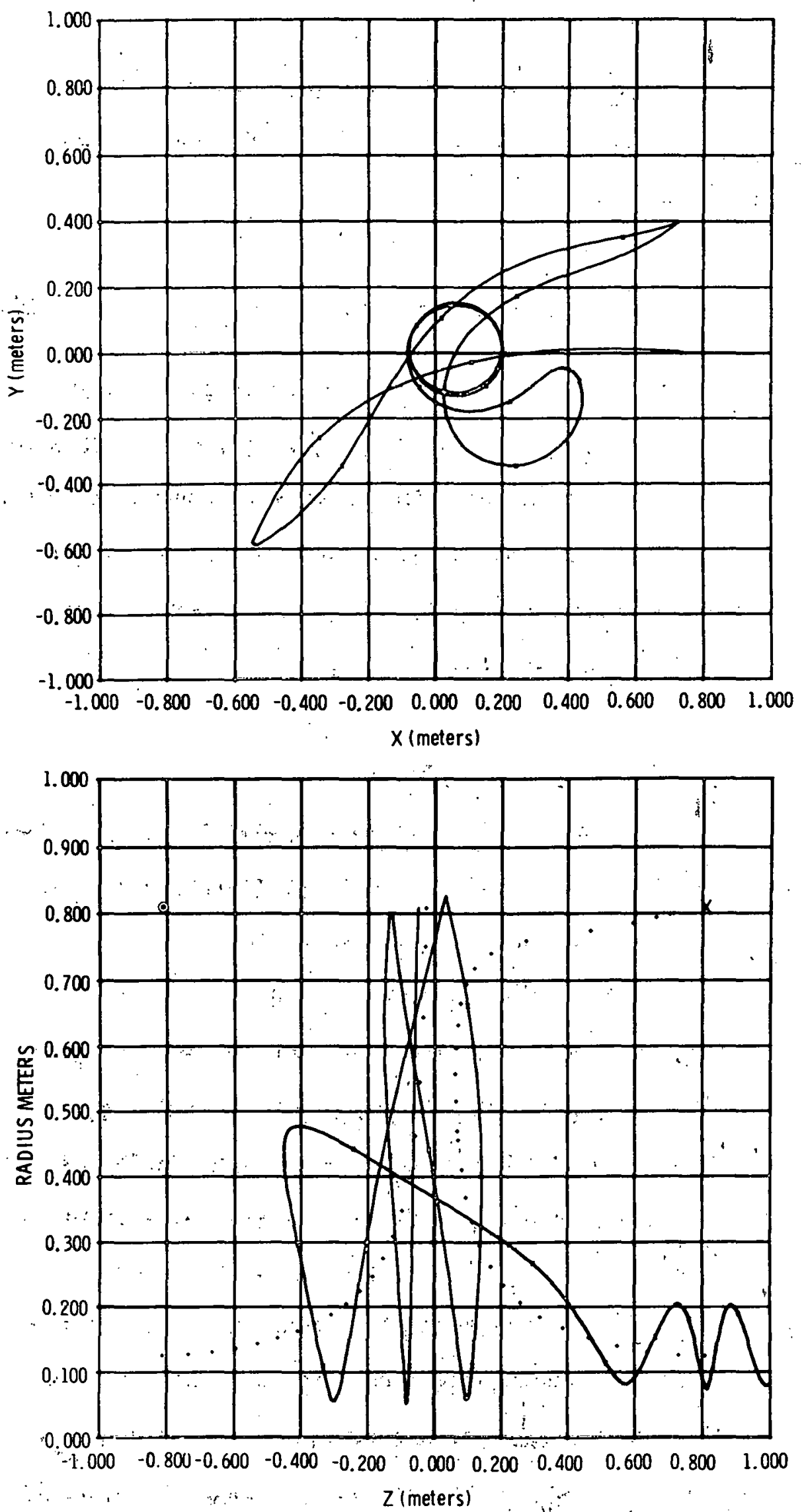

FIGURE 6 
$p_{r}=p_{z}=0$, when the total electron energy is in angular momentum. The surfaces which separate the accessible and inaccessible regions of the cusp are obtained by solving

$$
\frac{\mathrm{P}_{\mathrm{o}}}{\mathrm{r}}+\mathrm{eA} \mathrm{\phi}_{\phi}(\mathrm{r}, \mathrm{z})=\mathrm{mc}[\mathrm{U}(2+\mathrm{U})]^{1 / 2} \text {. }
$$

These lines are plotted in Fig. 7a,b for the trajectories shown in Figs. 5b and 6b, respectively. The loss cones are obvious. Therefore, only those electrons which are injected initially with angular momentum sufficientìy small to strike the target will ever strike the target. For the large aspect ratio (injection radius/pellet radius) of a reactor, this gives a very poor energy deposition efficiency.

\section{Randomizing Reflections at Mirror Points}

A physical mechanism which will change the electron angular momentum and provide the reflection (via virtual cathode formation) needed to plug the loss cones is the introduction of thin foils

(thickness « electron range) in the mirror regions. ${ }^{3}$ It could be argued that since the electrons' mechanical angular momentum is highest in these regions, that the net effect of randomizing reflections would be to preferentially destroy angular momentum as well as randomize the electron motion in the cusp, so that nearly all of the injected electrons could strike the target after several transits across the cusp. Unfortunately, neither of these may actually occur. While it is true that the randomizing reflections in the mirror regions will tend to lower the mechanical angular momentum, the conserved quantity between reflections is the canonical angular momentum, and since the randomizing 

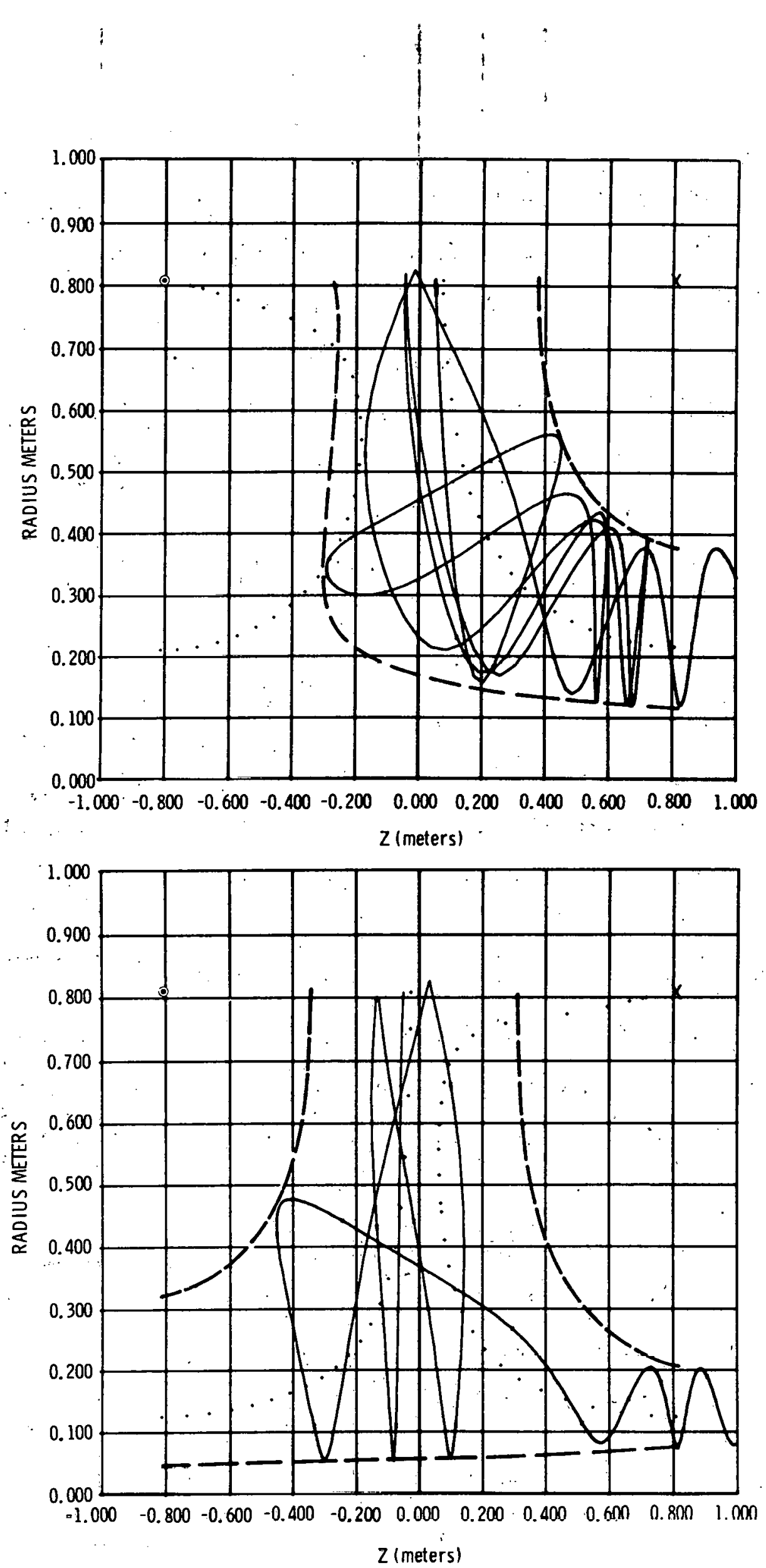

FIGURE 7 
reflection occurs at some finite value of $A_{\phi}(r, z)$, the cusp field itself puts a lower limit on the extent to which angular momentum can be reduced. In fact, if the cusp field is large enough, the canonical angular momentum may change very little when randomizing reflections are included, as will be shown to be the case in calculations performed for experiments reported to date. Also, between reflections the electrons move along trajectories governed by their equations of motion, so that the subsequent transits of the cusp do not possess a true random nature.

\section{E. Summary of Reported Soviet Experimental Results}

The most detailed reports of experimental results of injecting a ring current of electrons into a cusp were made by the Kurchatov group at the Albuquerque meeting in November $1975^{4}$ and at Berchtesgarten (FRG) in october 1976. ${ }^{5}$ The accelerator used was URAL (200-300 keV, $50 \mathrm{kA}, 60-70 \mathrm{~ns}, 500 \mathrm{~J})$ configured with coaxial anode and cathodes to produce a radially converging disk beam. They reported:

i) The injected beam burned a $1.5-4 \mathrm{~mm}$ wide hole in the 12-15 $\mathrm{mm}$ thick aluminum anode foil, with a calculated $5 \%$ beam loss in a single transit of the foil. The beam did not make multiple passes (>5-10) through the anode foil.

ii) The cusp coils were placed either 2 or $4 \mathrm{~cm}$ apart and had a diameter of $8 \mathrm{~cm}$. The maximum field strength (at $4 \mathrm{~cm}$ separation) was given as $13 \mathrm{kG}$ at the cusp throat and $6 \mathrm{kG}$ at the mirror points. (Using Eqs. 2 and 3, the total current flowing in each -coil was $80 \mathrm{kA}$. ) 
iii). A. steel aperture $1 \mathrm{~mm}$ wlde was placed over the anode foil to test effects of moving the injection plane with respect to the center of the cusp. When the injection plane was shifted $2 \mathrm{~mm}$, the calorimeter at the far mirror point dropped to $40-50 \%$ of the readings at the near calorimeter." Background pressure was varied from $5 \times 10^{-4}-1$ Torr with no effect, demonstrating the collisionless nature of the injected electrons.

iv) Targets of $8 \mathrm{~mm}$ diameter collected about $30 \%$ of the injected beam energy, relatively independent of background pressure and magnetic field variations, although $x$-ray pinholle photographs showed more nonuniform irradiation at higher pressures.

Among.the conclusions which were presented are:

(a) Since the cusp field was strong enough to prevent electrons injected more than $1.3 \mathrm{~mm}$ away from the center plane of the cusp from crossing the center plane, result (iii) showed that a significant number of electrons were trapped in the cusp: It was further assumed that "the captured electrons completely forget the prehistory of their movement," and thus the number of captured electrons incident on both calorimeters in the mirror regions is the same. This amounts to about $60 \%$ capture efficiency of injected electrons for symmetrical injection.

(b) Result (iv) "indicates scattering of the beam. The radiation symmetry and high level of energy transfer effectiveness to the target in a case of scattering of the beam which has entered the trap can be explained by the fact that the magnetic field used is a good trap for relativistic electrons; the equatorial slit of this trap is blocked 
by electrical and magnetic fields in the diode, and the loss area through the plugs is small. In this trap the relativistic electrons entering it should accumulate in the form of a cloud in the region of the neutral pole."

\section{F. Analysis of the Kurchatov Results}

The analytical and numerical techniques developed in sections $B-D$ will be used to evaluate the results reported to date, and alternate interpretations will be presented. The basic thesis of this section is that the experimental results obtained so far do not represent a valid test of the cusp fusion concept. Evaluation of the concept in a more generalized context will be given in section $G$.

Calculations have been performed for a cusp field given by Eqs. 1-3, with $I=80 \mathrm{kA}, r_{c}=4 \mathrm{~cm}, z_{c}=2 \mathrm{~cm}$, which gives $B_{r}=13 \mathrm{kG}$ at $r=r_{c}, z=0$, and $B_{z}= \pm 8 k G$ at $z=\mp z_{c}$. The electron energy was taken to be $300 \mathrm{keV}$, and a beam temperature of $3.6 \mathrm{keV}$ was obtained by calculating the mean square scattering angle ${ }^{6}$ of the beam through a $12 \mu \mathrm{m}$ thick aluminum foil. Electrons were injected into the cusp at the throat from $z=-2 \mathrm{~mm}$ to $z=2 \mathrm{~mm}$. The optimistic assumptions of a specularly reflecting surface at the cusp throat, and randomizing reflections at the mirror planes were included in the calculations. Flgure 8 shows the resulting trajectory for an electron injected with $z=1 \mathrm{~m}$. Clearly the cusp fields are so strong that not only is the electron restricted to the $z>0$ side of the cusp, but the randomizing reflections at the mirror plane are not even sufficient to allow the trajectory to get outside the initial accessible region. This is an 


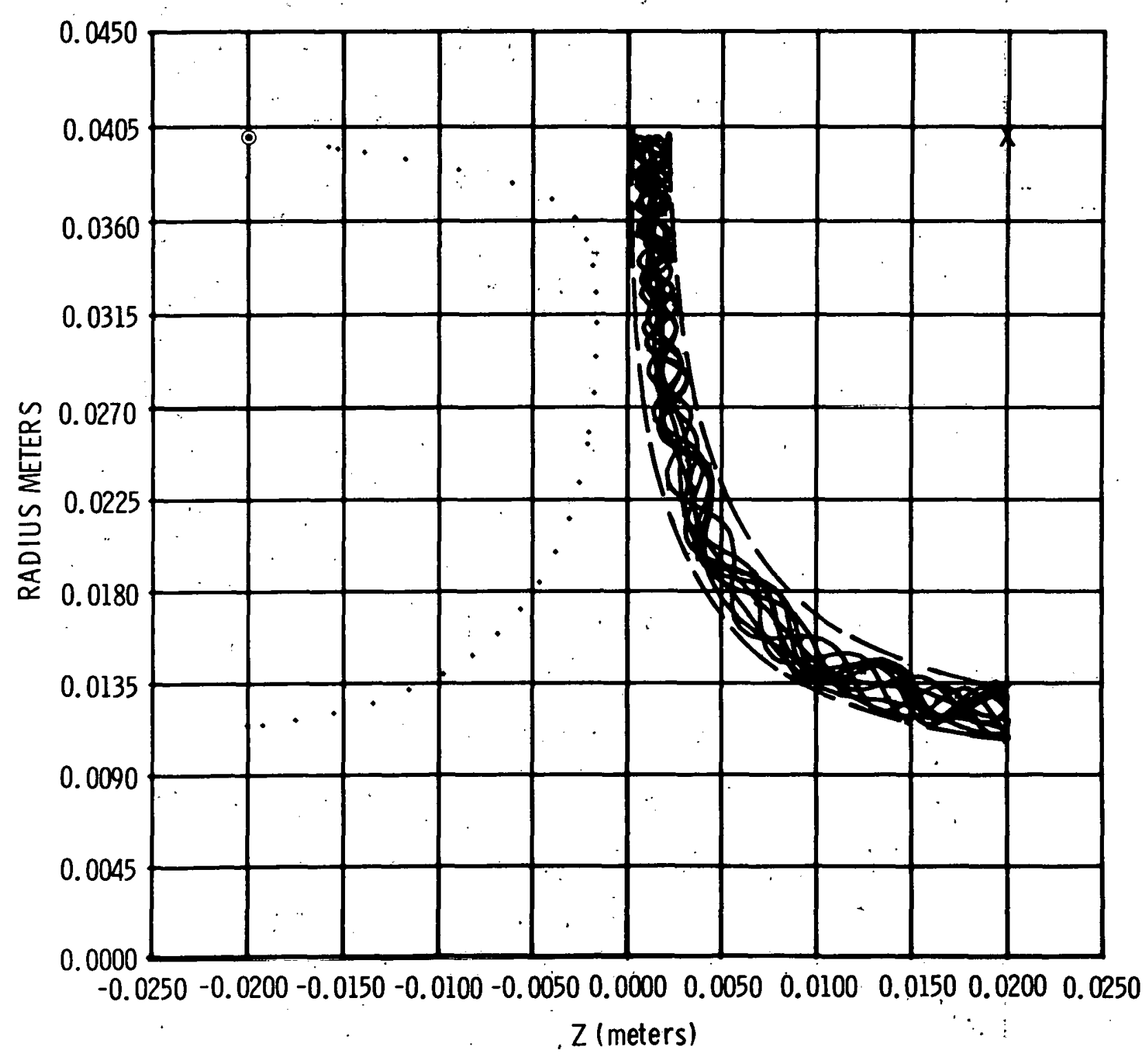

FIGURE 8 
example of the canonical angular momentum being totally dominated by the magnetic vector potential as discussed in section $D$. In order to minimize this effect, Fig. 9 shows an electron trajectory with injection at $\mathrm{z}=0$, where $A_{\phi}=0$ and the initial canonical angular momentum is just the mechanical angular momentum. Although the trajectory now shows some effects of reducing the angular momentum from its initial value, it still does not intercept the target region of radius $4 \mathrm{~mm}$. Note that rather than electrons accumulating near the origin, the opposite occurs.

Clearly the magnetic fields used have been too strong. A general mule of thumb which has proved useful in generating the plots shown in Figs. 4-7 is to keep the coil current near the Alfvén critical current, $I_{A}=17 \mathrm{By} \mathrm{kA}$ (35 $\mathrm{kA}$ for $700 \mathrm{keV}$ electrons), to allow the electrons access to both sides of the cusp and make mechanical angular momentum more important as compared to $A_{\phi}$.

An alternative interpretation of the reported results which arises from this evaluation is that the beam may not be fully. current neutralized on injection. The subsequent diamagnetic action of the injected beam causes a substantial number of electron's to be injected into a relatively field free region. These electrons are free to pass near the origin, restricted only by their mechanical angular mamentum. Since an electron injected such that, all of its "temperature" is in $v_{\phi}$ will have an angular momentum pinching limit of $r=6 \mathrm{~mm}$ for the above parameters used for the Kurchatov experiments, it is easy to explain the observed $30 \%$ energy deposition reported. If electrons were injected into a relatively field-free region, the aspect ratio (injection radius/pellet radius) of 10:1 was not large enough for angular momentum to be important. 


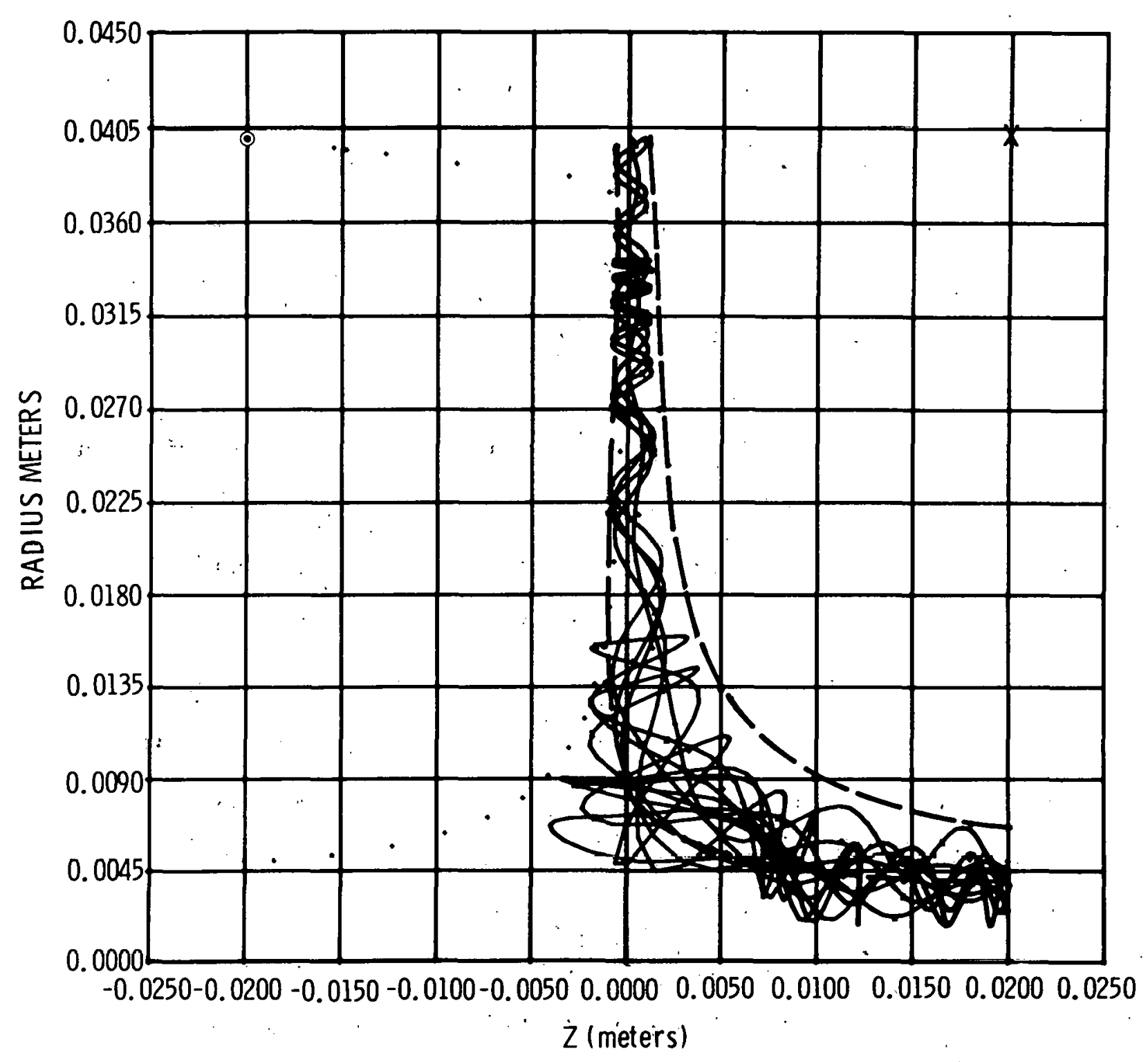

FIGURE 9 
As a result of this discussion, it seems' that the experiments reported to date do not represent a valid test of the cusp concept.

\section{G. Theme and Variations}

The strength of the cusp field must be carefully chosen if randomizing reflections at the mirror planes are to work in reducing angular momentum enough to allow the electron to strike the target. However, reducing the field strength increases the size of the loss cones, requiring larger mirror plane stoppers.

A possible improvement on the charge-neutral, current neutral injection scheme proposed to date, and investigated here, is to allow the beam self-fields to play a role. Those beam electrons which are injected diamagnetically into an essentially field-free region may tend to accumulate near the target. In this situation, we can envision a cloud of electrons trapped in a field free region by the cusp fields, so that angular momentum is all we have to contend with. Rudakov ${ }^{4}$ has, given an expression for efficient energy deposition in this case which we will now derive to examine the assumptions made.

Approximating the outer electron cloud boundary by a sphere at radius $R_{c}$, and designating the target pellet radius by $r_{p}$, the solid angle subtended by the target at $\dot{R}_{c}$ is given by

$$
\Omega_{t}=2 \pi\left(1-\cos \theta_{t}\right)=2 \pi\left[1-\left(1-r_{p}^{2} / R_{c}^{2}\right)^{1 / 2}\right] .
$$

where $\theta_{t}$ is the planar half angle subtended by the target at $R_{c} \cdot$ The crucial assumption is that the electrons are randomly scattered at $R_{c}$, and travel ballisticaily across the field-free region. 
This provides an isotropic angular distribution of electrons scattered back into the field-free region. The probability that in a single reflection an electron is scattered into the solid angle occupied by the target is

$$
P_{t}=\frac{\Omega_{t}}{2 \pi}=1-\left(I-r_{p}^{2} / R_{c}^{2}\right)^{1 / 2}
$$

If $r_{p} \approx R_{c}, P_{t} \approx I$ and the deposition will be efficient. However, for practical schemes $r_{p} \ll R_{c}$, so that the probability is low that an electron will hit the target on a single pass, and many passes are required to increase the net probability:

$$
\mathrm{P}_{N}=\mathrm{NP}_{t} \leq \frac{\mathrm{N}}{2}\left(\frac{r_{p}}{R_{c}}\right)^{2}
$$

Estimating the number of bounces during the lifetime $T$ of the electron in the cloud to be the order of $c \tau / R_{c}$, and requiring $P_{N} \approx 1$ for efficient deposition gives Rudakov's expression

$$
R_{c} \leq r_{p}\left(\frac{c \tau}{2 r_{p}}\right)^{I / 3}
$$

The difficulty with this calculation comes in trying to justify the crucial assumption made after Eq. (16). 'In order for the electrons to remain trapped for a reasonable portion of the pulse duration, the reflection mechanism at $R_{c}$ must be nearly specular. In other words, the electron Larmor radius at $R_{c}$ must be much smaller than any "scattering length" which deflects the electron from its collisionless trajectory. Otherwise, the electrons will diffuse rapidly across the magnetic boundary and will not be trapped. 
If we use a specular reflecting boundary, condition, angular momentum is conserved, and only those electrons which enter the field-free region with trajectories that intercept the target on the first pass can deposit any energy. If its transverse velocity is too large, the electron will continue to miss the target every pass. The condition that an electron will strike the target (replacing Eq. 19) is found to be

$$
v_{r} /|\vec{v}| \geq \cos \theta_{t}=\left(1-r_{p}^{2} / R_{c}^{2}\right)^{1 / 2},
$$

or $R_{c} \leq r_{p}|\vec{v}| / v_{\perp}$. This represents a very stringent condition on $R_{c}$ and the reader will note that it is simply a statement of conservation of angular momentum.

Therefore, either the geometry of the reflecting surface must be such as to randomize the electrons, or the electron angles of incidence at the boundary must be randomized before they reach the specularly reflecting boundary. Since a concave inward surface (such as the sphere assumed above) will always tend to return the electron to, smallei radii, and a convex inward surface (such as the undisturbed cusp flelu) lias the opposite effect, it is difficult to see how the surface geometry wi 1.1. help increase the deposition efficiency (unless a corrugated spherical surface can be generated). This leaves us with trying to randomize the electron angles of incidence before specular reflection takes place. This is also a difficult task, sluce if volume scattering is invoked, it is irrelevant to randomize the electron angles of incidence at the cloud boundary because the electron will be rescattered on its way back toward the target. Such a uniform volume scatterer. would do little to increase the deposition on the pellet. 
However, if the scattering probability increases toward the center of the trapping region, a beneficial effect occurs, since the probability that a scattering event will occur is greatest when the electron is at its closest distance of approach, where all its energy is in angular momentum, allowing it to get closer to the target on successive passes. In fact, the expanding corona from a target pellet may naturally provide some of this effect.

As a final variation, consider the possibility of current-neutral injection into an imploding cusp. This is probably not an encouraging approach (aside from stability of the imploding liner) because the time scale for the cusp implosion is: of course much longer than the beam pulse length. Therefore, the beam would have to be injected near the end of the cusp implosion, and the cusp throat would then look like a magnetic mirror to the incoming beam, which would prevent most of the beam from entering the cusp.

\section{H. Conclusions}

In this report, an attempt has been made to understand some aspects of using magnetic cusps for electron beam-driven inertial confinement fusion. It has been found that the experimental results reported to date do not provide a valid test of the concept, since angular momentum limits were not important for the geometry used. To explain the observed deposition, it seems necessary to invoke some beam diamagnetic effects to allow beam electrons to reach the low field region near the center of the cusp. Generalizations of the basic cusp concept to include beam selffields and scattering seem to be necessary for a viable scheme. However, such a scenario has yet to be developed. 


\section{References}

1. H. Grad, Phys. Rev. Lett. $\underline{4}, 222$ (1960).

2. G. Schmidt, Phys. Fluids 2, 994 (1962).

3. D. A. Phelps, J. E. Rauch, and P. Korn, IEEE Trans. PS-4, 246 (1976).

4. E. I. Baranchikov, et al., Proceedings of the International Topical Conference on Electron Beam Research and Technology, SAND-76-5112, Vol. I, p. 284, November 1975.

5. E. I. Baranchikov, et al., Proceedings of the Sixth IAEA Conference on Plasma Physics and Controlled Thermonuclear Reactions, October 1976. Note added in proof: Yu. V. Koba, V. S. Pen'kina, L. I. Rudakov, A. D. Sukhov, and E. Z. Tarumov, Sov. Tech. Phys. Lett. 2 , 120 (1976), contains essentially the same information.

6. R. D. Birkhoff, "The Passage of Fast Electrons Through Matter," in Handbuch der Physik, bd 34 (Springer, Berlin, 1958), p. 53 ff. 
Distribution:

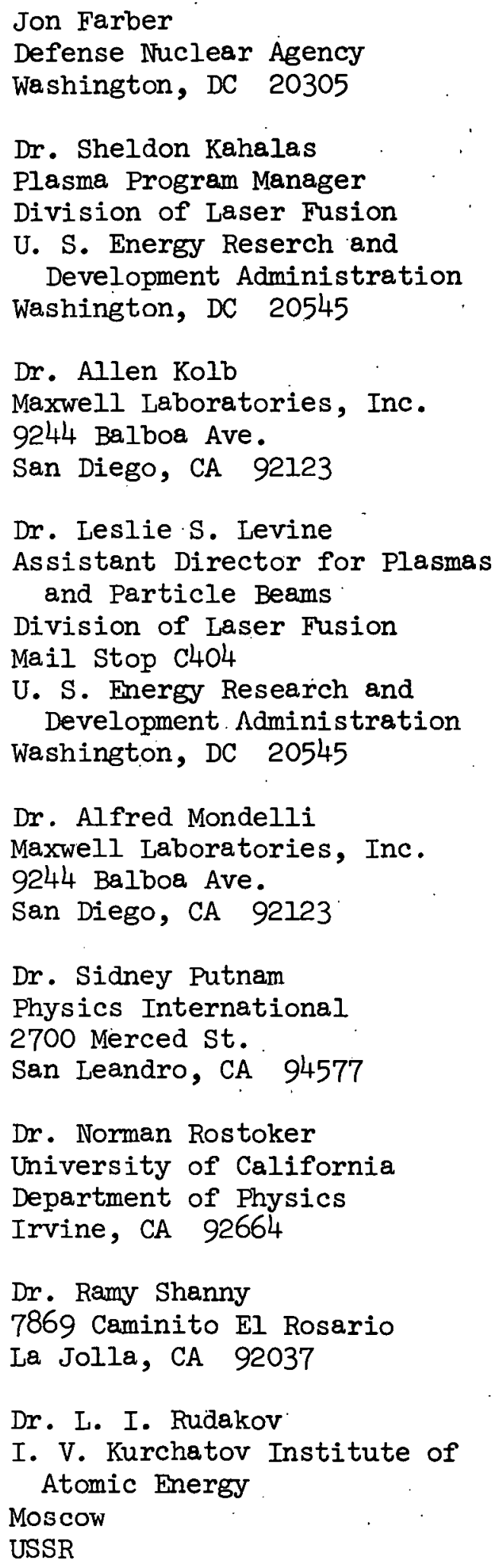

5000. A. Narath

$$
\begin{array}{lll}
\text { Attn: } & 5100 & \text { J. K. Galt } \\
& 5400 & \text { A. W. Snyder } \\
5700 & \text { J. H. Scott } \\
& 5800 & \text { R. S. Claassen }
\end{array}
$$

5200 E. H. Beckner

Attn: 5210 J. B. Gerardo

5240 G. Yonas

$$
5230 \text { M. Cowan }
$$

5241 J. R. Freeman

5241 L. Baker

5241 K. D. Bergeron

5241 M. J. Clauser

5241 A. V. Farnsworth, Jr.

5241 G. R. Hadley

5241 C. L. Olson

5241 J. W. Poukey

5241 J. P. Quintenz

5241 J. R. Stark

5241 M. A. Sweeney

5241. M. M. Widner

5241 T. P. Wright (20)

5242 A. J. Toepfer

5242 W. B. Boyer

5242 J. Chang

5242 R. J. Leeper

5242 L. P. Mix

5242 F. C. Perry

5243 G. W. Barr

5243 M. Aker

5244 G. W. Kuswa

5244 E. J. T. Burns

5244 S. A. Goldstein

5244 C. W. Mendel, Jr.

5244 F. A. Miller.

5244 J. N. Olsen

5245 T. H. Martin

5245 D. L. Johnson

5245 D. H. McDaniel

5245 E. L. Neau

5245 J. P. VanDevender

5246 K. R. Prestwich.

5246 M. T. Buttram

5246 J. J. Ramirez

5246 G. J. Rohwein

8266 E. A. Aas (2)

3151 W. L. Gamer (3) For ERDA/TIC Public Release

3141 Central Tech. Files (4)

3171-1 R. P. Camphe1.1. (25) For ERDA/TIC 